\title{
Immunocytochemical localization of monoamine oxidase type $B$ in rat liver
}

\author{
Y.H. Huang, M. Jiang, B.Y. Fu
}

Department of Gastroenterology, The First Affiliated Hospital, China Medical University, Shenyang, China

(C)2008 European Journal of Histochemistry

We used an immunohistochemical method to examine the localization of monoamine oxidase type $B(M A O B)$ in rat liver. At the light microscopic level, MAOB was highly expressed in rat liver. It was intense around portal area, and weak around central area. All the hepatocytes examined had MAOB immunoreactivity. For the first time, using a doublelabeling immunofluorescence histochemical method for laser microscopy, we report that no MAOB is found in endothelial cells, hepatic stellate cells, or Kupffer's cells. When examined under transmission electron microscopy, MAOB was localized to the mitochondrial outer membrane of hepatocytes. No apparent localization of MAOB was found in the rough endoplasmic reticulum, the crystal membrane of mitochondria, the nuclear envelope, or the plasma membrane.

Key words: Monoamine oxidase, liver, hepatocyte, endothelial cell, hepatic stellate cell, kupffer's cell, mitochondria, double-labeling immunofluorescence histochemistry, electron microscopic immunohistochmistry

Correspondence: Jiang Min,

Department of Gastroenterology,

The First Affiliated Hospital, China Medical University, Shenyang 110001, China

Tel.: +86-24-8328-2199.

Fax: +86-24-2326-7030

E-mail: jiangminhyh@hotmail.com

Paper accepted on December 20, 2007

European Journal of Histochemistry

2008; vol. 52 issue 1 (Jan-Mar): 11-18
$\mathrm{M}$ onoamine oxidase (MAO) is a mitochondrial flavoprotein enzyme, which oxidatively deaminates a wide range of monoamines. This enzyme is found in two functionally distinct forms, MAO type A (MAOA) and type B (MAOB) (Bach et al., 1988; Berry et al., 1994).

Previous studies have shown that MAOA and MAOB activities are present in homogenates of rat liver (Schnaitman et al., 1967; Lewinsohn et al., 1978; Lenzen et al., 1983). Enzyme histochemistry (Ryder et al., 1979; Egashira and Waddell, 1984; Uchida and Koelle, 1984) and quantitative enzyme radioautography (Saura et al., 1992) have shown that both MAOA and MAOB are expressed in rat liver at the light microscopic level. Only two papers (Shannon et al., 1974; Muller and Lage, 1977) examine MAO localization at the electron microscopic level, but they use an enzyme histochemical method. All the above methods are not sensitive nor specific. To our knowledge, there are only four published papers that use an immunocytochemical technique (Thorpe et al., 1987; Rodriguez et al., 2001; Ramonet et al., 2003; Sivasubramaniam et al., 2003). But they examine MAOA and MAOB localization in human liver at light microscopic level.

In this paper, using rabbit anti-MAOB polyclonal antibodies, for the first time, by immunohistochemical method, we examined the cellular and subcellular localization of MAOB in rat liver at the light and electron microscopic level, to gain further insight into the functional role of this enzyme in the rat liver.

\section{Materials and Methods}

\section{Animals}

Male Sprague-Dawley rats $(n=6,180-200 \mathrm{~g})$ were obtained from China Medical University. All experiments were carried out according to the Guidelines for Animal Experimentation at China 
Medical University. They were housed in temperature-, humidity-, and light-controlled rooms $\left(23 \pm 1^{\circ} \mathrm{C}, 50 \pm 5 \%\right.$, and light on from 8:00 a.m. to 8:00 p.m.), and had free access to standard food and tap water.

\section{Primary antibodies}

Sheep antibodies against rat albumin (Bethyl Laboratories, Montgomery, USA), mouse antibodies against human CD34, smooth muscle-specific $\alpha$-actin (SMSA), and CD68 (DAK0, Carpinteria, CA, USA) were used. These antibodies have been previously characterized (Cizkova et al., 2005; Chedid et al., 2004).

MAOB purified from bovine liver mitochondria was used to generate a rabbit anti-MAOB antiserum (Sagara and Ito, 1982). The specificity of this antiserum is described in the discussion.

\section{Preabsorption test}

Based on Ma and Ito (2002), cDNA of rat liver MAOB was prepared in a hexa-histidine tagged form, expressed in Escherichia coli, and purified using a HisTrap chelating column. The recombinant protein was used for a preabsorption test as follows. Thirty milligrams of rat MAOB protein was added to $1 \mathrm{~mL}$ of diluted anti-MAOB antiserum $(1: 50,000)$, and the mixture $(0.5 \mathrm{mM}$ MAOB) was incubated for 24 hours at $4^{\circ} \mathrm{C}$. Using this preabsorbed antiserum, sections of the liver were processed for immunofluorescence histochemistry as described below.

\section{Double-labeling immunofluorescence histochemistry}

Three rats were anesthetized with sodium pentobarbital $(60 \mathrm{mg} / \mathrm{kg}$ body weight, i.p.) and perfused through the ascending aorta with $150 \mathrm{~mL}$ of 0.01 $M$ phosphate-buffered saline (PBS; $\mathrm{pH} 7.4$ ), followed by $300 \mathrm{~mL}$ of a fixative solution containing $4 \%$ paraformaldehyde in $0.1 \mathrm{M}$ phosphate buffer ( $\mathrm{PB} ; \mathrm{pH}$ 7.4). The livers were dissected and placed in the same fixative solution for 24 hours, and then immersed in PB containing $15 \%$ sucrose. $10 \mu \mathrm{m}$ thick sections were cut using a cryostat (Cryocut 1800, Reichert-Jung, Heiderberg, Germany), and collected in PBS.

Double-labeling of MAOB and each of liver cell markers was performed as follows. Sections were incubated in the following solutions: (1) $0.3 \%$ Triton X-100 in PBS for 1 hour; (2) $1 \%$ bovine serum albumin (BSA) in PBS for 1 hour; (3) a mixture of primary antisera: rabbit antiserum against MAOB (dilution, 1:5,000) combined with one of the four antisera: sheep anti-albumin antiserum (dilution, 1:2,000), mouse anti-CD34 antiserum (dilution, 1:200), mouse anti-SMSA antiserum (dilution, 1:200), and mouse anti-CD68 antiserum (dilution, 1:200) for 48 hours; and (4) a mixture of secondary antisera: Alexa Fluor 488conjugated goat anti-rabbit immunoglobulin $G$ (IgG) (dilution, 1:200; Molecular Probes, Eugene, Oregon, USA, ) combined with one of the two antisera: Cy3-conjugated donkey anti-sheep IgG (dilution, 1:200; Chemicon, Temecula, CA, USA, ), and Cy3-conjugated donkey anti-mouse IgG (dilution, 1:200; Chemicon) for 2 hours. Finally, MAOB was labeled with Alexa Fluor 488 (green), Albumin, CD34, SMSA, and CD68 were labeled with Cy3 (red). The stained sections were observed under a confocal laser scanning microscope (LSM510, Zeiss, Jena, Germany).

\section{Electron microscopic immunohistochemistry}

Three rats were anesthetized with sodium pentobarbital (60 mg/kg body weight, i.p.) and perfused through the ascending aorta with $150 \mathrm{ml}$ of 0.01 M PBS, followed by $300 \mathrm{ml}$ of a fixative solution containing $4 \%$ paraformaldehyde, $0.3 \%$ glutaraldehyde, and $0.2 \%$ picric acid in $0.1 \mathrm{M} \mathrm{PB}$. The livers were dissected and placed in another fixative solution containing $4 \%$ paraformaldehyde and $0.2 \%$ picric acid in PB for 24 hours. $30 \mu \mathrm{m}$ thick sections were cut using a vibratome (DTK3000, Dosaka, Kyoto, Japan), and collected in PBS.

For MAOB staining, an immunoperoxidase technique using avidin-biotin-peroxidase complex (Hsu et al., 1981) was performed. Sections were incubated in the following solutions: (1) $0.001 \%$ trypsin (type III, Sigma, St. Louis, MO, USA) in PBS for 5 minutes; (2) $5 \%$ normal goat serum in PBS for 1 hour; (3) rabbit anti-MAOB antiserum (dilution, 1:5,000) for 48 hours; (4) biotinlyted goat anti-rabbit IgG (dilution, 1:1,000; Vector, Burlingame, USA) for 2 hours; (5) avidin-biotinperoxidase complex (dilution, 1:1,000; Vector) for 2 hours; and (6) $0.025 \%$ 3,3'-diaminobenzidine (Dojindo, Kumamoto, Japan), 0.6\% nickel ammonium sulfate (Nacalai Tesque, Kyoto, Japan), and $0.0075 \%$ hydrogen peroxide $\left(\mathrm{H}_{2} \mathrm{O}_{2}\right)$ in $0.05 \mathrm{M}$ trizma hydrochloride (Tris- $\mathrm{HCl}$ ) buffer $(\mathrm{pH} 7.6)$ for 5 minutes. The stained sections were fixed with 
$1 \%$ osmium tetroxide (Nacalai Tesque, Kyoto, Japan) for 1 hour, dehydrated and flat-embedded in epoxy resin (Luveak-812, Nacalai Tesque, Kyoto, Japan). Small areas were trimmed from the embedded section and cut on an ultramicrotome (Ultracut UCT, Leica, Heiderberg, Germany). Ultrathin sections were collected on grids (200, Cu, VECO, Eerbeek, Holland), stained with $2 \%$ uranyl acetate (Merck, Darmstadt, Germany) for 20 minutes and lead stain solution (Sigma-Aldrich Japan, Tokyo, Japan) for 5 minutes, and examined with a transmission electron microscope $(\mathrm{H}-$ 7100TE, Hitachi, Tokyo, Japan).

\section{Results}

\section{Preabsorption test}

Preabsorption of the anti-MAOB antiserum with rat recombinant $\mathrm{MAOB}$ protein $(0.5 \mathrm{mM})$ eliminated the immunostaining (Figure $1 \mathrm{~B}$ ).

\section{Immunofluorescence histochemistry}

Figure $1 \mathrm{~A}$ shows the localization of $\mathrm{MAOB}$ in rat liver. In all the samples examined, MAOB was highly expressed in the rat liver. It was intense around portal area, and weak around central area.

\section{Double-labeling immunofluorescence histochemistry}

Figure 2 shows the cellular localization of MAOB in rat liver. All of hepatocytes that were labeled for albumin were also stained for MAOB (Figure $2 \mathrm{~A}, \mathrm{~B}, \mathrm{C}$ ). No MAOB was found in endothelial cells (cells that were labeled for CD34, Figure 2 D, E, F), hepatic stellate cells (cells that were labeled for SMSA, Figure $2 \mathrm{G}, \mathrm{H}$, I), or Kupffer's cells (cells that were labeled for
CD68, Figure $2 \mathrm{~J}, \mathrm{~K}, \mathrm{~L}$ ). The staining was primarily cytoplasmic, and could be seen to be punctate in suitable sections (Figure $2 A, D, G, J$ ). This appearance reflects the mitochondrial localization of MAOB.

\section{Electron microscopic immunohistochemistry}

Figure 3 shows the subcellular localization of $M A O B$ in rat liver. MAOB immunoreactivity was found on the mitochondrial outer membrane of hepatocytes. No apparent localization of MAOB was found in the rough endoplasmic reticulum, the crystal membrane of mitochondria, the nuclear envelope, or the plasma membrane.

\section{Discussion}

In this report, using an immunohistochemical method, we presented results which localized MAOB in rat liver at the light and electron microscopic level. By light microscopy, we found that MAOB was highly expressed in rat liver. These are in general agreement with the findings of other researchers (Schnaitman et al., 1967; Lewinsohn et al., 1978; Ryder et al., 1979; Lenzen et al., 1983; Egashira and Waddell, 1984; Uchida and Koelle, 1984; Thorpe et al., 1987; Saura et al., 1992; Rodriguez et al., 2001; Ramonet et al., 2003; Sivasubramaniam et al., 2003), which all show that both MAOA and MAOB are expressed in rat liver. But most of these studies use enzyme histochemistry, quantitative enzyme radioautography, spectrophotometric assay, and radiochemical assay. All of the above methods are neither sensitive nor specific, because no compounds show absolute isozyme specificity. All compounds described will interact

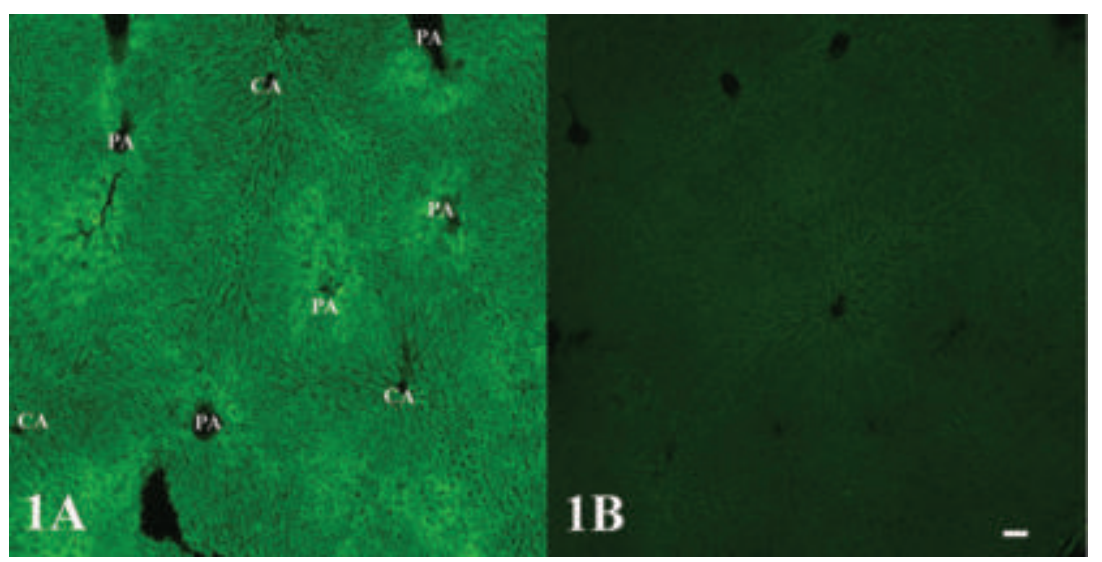

Figure 1. Preabsorption test. Sections of rat liver are processed for immunofluorescence histochemistry using non-absorbed anti-MAOB antiserum (A) and antiserum preabsorbed with rat recombinant MAOB protein (0.5 $\mathrm{mM}$; B). MAOB is highly expressed in rat liver. It is intense around portal area (PA), and weak around central area (CA) (A). Preabsorption eliminate the immunostaining (B). Original magnification $\times 4$. Bar $=100 \mu \mathrm{m}$. 

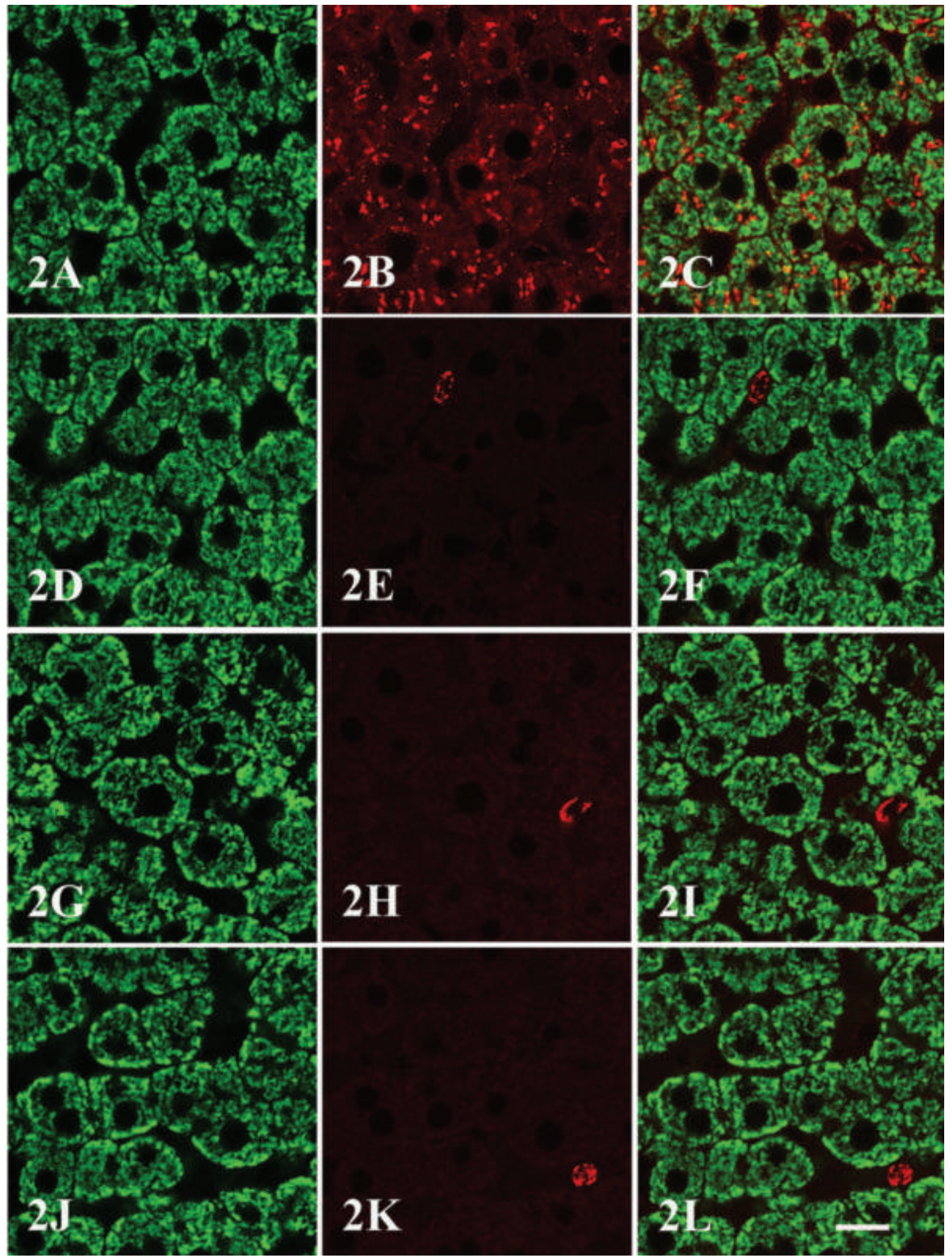

Figure 2. Cellular localization of MAOB in rat liver. (A, D, G, J) MAOB staining (green). (B) Albumin staining (red). (E) CD34 staining (red). (H) SMSA staining (red). (K) CD68 staining (red). (C, F, I, L) Superimposition of the images in panels A, D, G, J and B, E, H, K. Note that all of hepatocytes that are labeled for albumin are also stained for MAOB (A, B, C). No MAOB is found in endothelial cells (D, E, F), hepatic stellate cells (G, H, I), or Kupffer's cells $(J, K, L)$. The staining is primarily cytoplasmic, and could be seen to be punctate in suitable sections (A, D, G, J). Original magnification $\times 100$, Bar $=5 \mu \mathrm{m}$. 

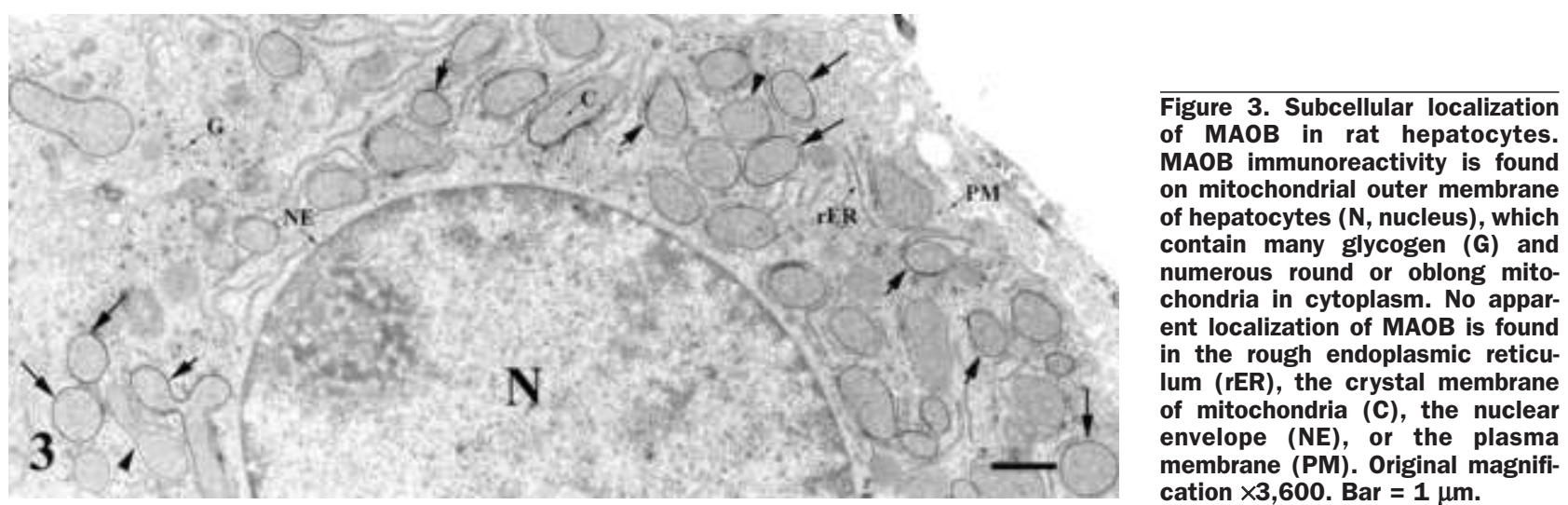

with both forms of MAO under favorable conditions (Berry et al., 1994), such as $10^{-3} \mathrm{M}$ pargyline and $10^{-4} \mathrm{M}$ deprenyl, which inhibit both MAOA and MAOB (Ryder et al., 1979; Lewinsohn et al., 1980). 5-hydroxytryptamine (5-HT), long considered to be a substrate only for MAOA, is mainly $(70 \%)$ deaminated by $\mathrm{MAOB}$, but with a higher $\mathrm{K}_{\mathrm{m}}$ (Stenstrom and Lundquist, 1990). In addition, most of them use tryptamine or tyramine (TYR) as substrates, which are substrates of both MAOA and MAOB. They can not distinguish between MAOA and MAOB. In the present study, we localized MAOB in the rat liver using antiserum against MAOB purified from the bovine liver mitochondria. Specific cross-reactivity of the anti-MAOB antiserum with rat MAOB was demonstrated as follows: (1) Solubilized mitochondria of the rat liver were subjected to immunoprecipitation with the anti-MAOB antiserum, and then the precipitate was analyzed by sodium dodecyl sulfate-polyacrylamide gel electrophoresis, showing a single band corresponding to a molecular mass that was calculated from cDNA of rat liver MAOB (Ito et al., 1988; Sagara and Ito, 1982). (2) Immunohistochemical analysis showed that the anti-MAOB antiserum did cross react with $\mathrm{MAOB}$ but not $\mathrm{MAOA}$ in the rat brain (Arai et al. 2002). Furthermore, we showed in the present study that preabsorption of the anti-MAOB antiserum with rat recombinant MAOB protein eliminated the immunohistochemical staining. Altogether, these findings indicate that the immunohistochemical staining with the anti-MAOB antiserum show the localization of $M A O B$ in the rat liver.

Our results showed that all the hepatocytes examined had MAOB immunoreactivity. It was intense around portal area, and weak around cen- tral area. The reason for uneven distribution may reflect a difference in supplying blood and oxygen. The hepatocytes around portal area, which are first exposed to the blood entering the lobule, oxidative respiration via the Krebs cycle may operate at a high level. The enzyme activity is higher. While the cells around the central area, which carry the blood leaving the lobule, the enzyme activity is lower.

In this study, we stained the hepatocytes, endothelial cells, hepatic stellate cells, and Kupffer's cells with antibodies against albumin, CD34, SMSA, and CD68. Because previous studies have shown that these four antibodies are the best maker for each cell type(Cizkova et al., 2005; Chedid et al., 2004). For the first time, using a double-labeling immunofluorescence histochemical method for laser microscopy, we reported that in rat liver, no MAOB immunoreactivity was found in endothelial cells, hepatic stellate cells, or in Kupffer's cells. Though there were reports that $\mathrm{MAOB}$ is localized on capillary endothelial cells of rat tongue ( $X u$ et al., 2002), hepatic stellate cells of rat liver (Muller and Lage., 1977), and Kupffer's cells of human peripheral blood (Seiler et al., 1995). In fact, at electron microscopic level, we also found no MAOB was expressed in endothelial cells, hepatic stellate cells, or in Kupffer's cells (data not show). These maybe due to a difference in experimental methods and tissues. The exact reason remains to be explored.

When examined under transmission electron microscopy, MAOB was localized to the mitochondrial outer membrane of hepatocytes. These results are in general agreement with those of Shannon et al. (1974) and Muller and Lage (1977). By 
enzyme histochemistry, Shannon et al. (1974) found MAO reaction products in mitochondrial outer compartment, the rough endoplasmic reticulum, and the nuclear membrane of unfixed guinea pig liver, Muller and Lage (1977) described that MAO was localized to the mitochondrial outer membrane, the rough endoplasmic reticulum, the crystal membrane of mitochondria, the nuclear envelope, and the plasma membrane of rat hepatocytes and hepatic stellate cells. Whereas, we did not find any apparent localization of MAOB in the rough endoplasmic reticulum, the crystal membrane of mitochondria, the nuclear envelope, nor the plasma membrane. The reason maybe due to a difference in experimental methods. They use an enzyme histochemical method, it is neither sensitive nor specific. We use immunohistochemical method, it is sensitive and specific.

$\mathrm{MAO}$ is enzyme that degrades a wide range of monoamines. The primary roles of MAO lie in the metabolism of exogenous amines and the control of neurotransmitter levels and intracellular amine stores (Billett, 2004). It is found in two functionally distinct forms, MAOA and MAOB. They differ in substrate preference, inhibitor specificity, tissue and cell distribution, immunological property, and apparent molecular weight (Berry et al., 1994). Under normal physioloical conditions, MAOA preferentially oxidases 5-HT and norepinephrine (NE), whereas MAOB oxidases phenylethylamine (PEA) preferentially, with dopamine (DA) and TYR being substrates for both isoenzymes. MAOA is inhibited by low concentrations of clorgyine, and MAOB by low concentration of deprenyl (Billett, 2004).

$M A O B$ is a typical intrinsic protein of the outer mitochondrial membrane. It is synthesized in cytoplasmic polysomes (Sagara and Ito, 1982). It has an uncleavable targeting signal and is inserted into the outer membrane without proteolytic maturation. The targeting signal is present within its carboxy-terminal 29 amino acid residues (Mitoma and Ito, 1992). It inserts into the membrane by ubiquitin (a 76-amino acid polypeptide), with energy provided by ATP (Zhaung and McCauley, 1989).

Biochemical and histochemical results indicate large differences of MAO characteristics between animal species as well as in varying tissues. It is considered that the specific localization of the two forms of MAO within different body tissues is of biological and clinical significance (Stenstrom and Lundquist, 1990; Billett, 2004). The expression of MAO in liver suggests that this enzyme may play an important role in liver. In the disease of hepatic encephalopathy (HE), the neueotransmitters, such as DA, 5-HT, NE, and $\gamma$-aminobutyric acid (GABA), the neurotransmitters metabolites, such as asparagines, glutamine, homovanillic acid, normetanephrine, 5-hydroxyindolacetic acid (5-HIAA), and indole-3-acetic acid (IAA) are increased in cerebrospinal fluid (Zieve, 1987). MAO is the main degradation enzymes of monoamine hormones and amine neurotransmitters, such as epinephrine (E), NE, 5-HT, and DA (Billett, 2004). From these, we speculate that MAO may play an important role in liver function and in liver diseases. This is supported by the work of Rao et al. (1993). Using radioenzymatic assay, Rao et al. (1993) measured activities of MAOA and $M A O B$ in autopsied brain tissue from cirrhotic patients who died in HE. They found the activities of both MAOA and MAOB were significantly increased in frontal cortex and caudate nucleus, and concluded that increased monoamine metabolism and subsequent modifications of monoaminergic synaptic function could contribute to the pathogenesis of HE. MAOB in liver has the ability to deaminate certain amines. In the process of deamination, ammonia are generated (Berry et al. 1994). This substance is normally removed by the hepatobiliary route and that in liver failure accumulate in the brain. This alters the expression of key astrocytic proteins in astrocytes of the brain. Recent molecular biological studies show an increased expression of several genes coding for neurotransmitter-related proteins in chronic liver failure. Such genes include those for MAOB, glutamine synthetase, and the so-called peripheraltype benzodiazepine receptors. In addition, expression of some neuronal proteins such as MAOA and neuronal nitric oxide synthase are modified. Activation of these systems may lead to alterations of monoamine and amino acid neurotransmitter function and changes in cerebral blood flow in chronic liver failure. Available evidence suggests that neurotransmission changes rather than brain energy failure are the primary cause of $\mathrm{HE}$ (Hazell and Butterworth, 1999). Up to now, the exact role of MAO in liver and the mechanism of the change of MAO activity in brain of liver diseases are not completely understood. Further research is required regarding this question. 


\section{Acknowledgments}

We thanks professor Ryohachi Arai (Department of Anatomy, Shiga University of Medical Science) and professor Akio Ito (Department of Chemistry, Faculty of Science, Kyushu University) to provide us anti-MAOB antibody.

\section{References}

Arai R, Karasawa N, Kurokawa K, Kanai H, Horiike K, Ito A. Differential subcellular location of mitochondria in rat serotonergic neurons depends on the presence and the absence of monoamine oxidase type B. Neuroscience 2002;114:825-35.

Bach AWJ, Lan NC, Johnson DL, Abell CW, Bembenek ME, Kwan SW, Seeburg PH, Shih JC. CDNA cloning of human liver monoamine oxidase $A$ and $B$ : Molecular basis of differences in enzymatic properties. Proc Natl Acad Sci USA 1988; 85:4934-8.

Billett EE. Monoamine oxidase (MAO) in human peripheral tissue. Neurotoxicology 2004;25:139-48.

Berry MD, Juorio AV, Paterson IA. The functional role of monoamine oxidase $A$ and $B$ in the mammalian central nervous system. Prog Neurobiol 1994;42:375-91.

Chedid A, Arain S, Snyder A, Mathurin P, Capron F, Naveau S. The immunology of fibrogenesis in alcohoilic liver disease. Archives of Pathology \& Laboratory Medicine 2004;128:1230-8.

Cizkova D, Mokry J, Micuda S, Osterreicher J, Martinkova J. Expression of MRP2 and MDR1 transporters and other hepatic markers in rat and human liver in WRL 68 cell line. Physiol Res 2005;54:419-28.

Egashira T, Waddell WJ. Histochemical localization of monoamine oxidase in whole-body, freeze-dried sections of mice. Histochem J 1984; 16:919-29.

Hazell AS, Butterworth RF. Hepatic encephalopathy: An update of pathophysiologic mechanisms. Proc Soc Exp Biol Med 1999;222:99-112.

Hsu SM, Raine L, Fanger $\mathrm{H}$. Use of avidin-biotin-peroxidase complex $(A B C)$ in immunoperoxidase techniques: a comparison between $A B C$ and unlabeled antibody (PAP) procedures. J Histochem Cytochem 1981;29:577-80.

Ito $A$, Kuwahara T, Inadome $S$, Sagara $Y$. Molecular cloning of a cDNA for rat liver monoamine oxidase B. Biochem Biophys Res Commun 1988; 157:970-6.

Lenzen S, Nahrstedt $\mathrm{H}$, Panten U. Monoamine oxidase in pancreatic islets, exocrine pancreas, and liver from rats. Characterization with clorgyline, deprenyl, pargyline, tranylcypromine, and amezinium. Naunyn-Schmiedeberg's Arch Pharmacol 1983;324:190-5.

Lewinsohn R, Bohm KH, Glover V, Sandler M. A benzylamine oxidase distinct from monoamine oxidase B-widespread distribution in man and rat. Biochem Pharmacol 1978;27:1857-63.

Lewinsohn R, Glover V, Sandler M. Development of benzylamine oxidase and monoamine oxidase $A$ and $B$ in man. Biochem Pharmacol 1980;29:1221-30.

$\mathrm{Ma} \mathrm{J}$, and Ito A. Tyrosine residues near the FAD binding site are critical for FAD binding and for the maintenance of the stable and active conformation of rat monoamine oxidase $A$. J Biochem 2002;131:107-11.

Mitoma $J$, Ito A. Mitochondrial targeting signal of rat liver monoamine oxidase $B$ is located at its carboxy terminus. J Biochem 1992;111:20-4.

Muller J, Lage CD. Ultracytochemical demonstration of monoamine oxidase activity in nervous and non-nervous tissue of the rat. $J$ Histochem Cytochem 1977;25: 337-48.

Ramonet D, Rodriguez M, Saura J, Lizcano JM, Romera M, Unzeta $M$, et al. Localization of monoamine oxidase $A$ and $B$ and semicarbazide-sensitive amine oxidase in human peripheral tissue. Inflammopharmacology 2003;11:111-7.

Rao VL, Giguere JF, Layrargues GP, Butterworth RF. Increased activities of MAOA and MAO B in autopsied brain tissue from cirrhotic patients with hepatic encephalopathy. Brain Res 1993;621:349-52.

Rodriguez MJ, Saura J, Billett EE, Finch CC, Mahy N. Cellular localization of monoamine oxidase $A$ and $B$ in human tissues outside of the central nervous system. Cell tissue Res 2001;304:215-20.

Ryder TA, Mackenzie ML, Pryse-Davies J, Glover V, Lewinsohn R, Sandler M. A coupled peroxidatic oxidation technique for the histochemical localization of monoamine oxidase A and B and benzylamine oxidase. Histochemistry 1979;62:93-100.

Sagara $Y$, Ito $A$. In vitro synthesis of monoamine oxidase of rat liver outer mitochondrial membrane. Biochem Biophs Res Common 1982;109:1102-7.

Saura J, Kettler R, Prada MD, Richards JG. Quantitative enzyme radioautography with 3H-Ro 41-1049 and 3H-Ro 19-6327 in vitro: localization and abundance of $\mathrm{MAO}-\mathrm{A}$ and $\mathrm{MAO}-\mathrm{B}$ in rat CNS, peripheral organs, and human brain. J Neurosci 1992;12:1977-99.

Schnaitman C, Erwin VG, Greenawalt JW. The submitochondrial localization of monoamine oxidase. An enzymatic marker for the outer membrane of rat liver mitochondria. J Cell Biol 1967;32:719-35.

Seiler N, Moulinoux JP, Havouis R, Toujas L. Characterization of amine oxidase activities in macrophages from human peripheral blood. Biochem Cell Biol 1995;73:275-81.

Shannon WA Jr, Wasserkrug HL, Seligman AM. The ultrastructural localization of monoamine oxidase (MAO) with tryptamine and a new tetrazolium salt, 2-(2'-benzothiazolyl)-5-styryl-3-(4'-phthalhydrazidyl) tetrazolium chloride (BSPT). J Histochem Cytochem 1974;22:170-82

Sivasubramaniam SD, Finch CC, Rodriguez MJ, Mahy N, Billett EE. A comparative study of the expression of monoamine oxidase-A and $B$ mRNA and protein in non-CNS human tissues. Cell Tissue Res 2003;313:291-300.

Stenstrom A, Lundquist I. Monoamine oxidase (MAO) A and B in pancreatic islets from the mouse. Biogenic Amines 1990;7:54755.

Thorpe LW, Westlund KN, Kochersperger LM, Abell CW, Denney RM. Immunocytochemical localization of monoamine oxidases $A$ amd $B$ in human peripheral tissues and brain. J histoem Cytochem 1987;35:23-32.

Uchida $E$, Koelle GB. Histochemical investigation of criteria for the distinction between monoamine oxidase $A$ and $B$ in various species. $\mathrm{J}$ Histochem Cytochem 1984;32:667-73.

$\mathrm{Xu}$ Y, Yoshitake K, Ito A, Arai R. Monoamine oxidase type B is localized to mitochondrial outer membranes in mast cells, Schwann cells, endothelial cells and fibroblasts of the rat tongue. Acta Histochem Cytochem 2002;35:417-22.

Zieve L. Hepatic encehpalopathy. In: Schiff L and Schiff E.R, eds. Diseases of the liver. Lippincott Press, Philadelphia, 1987, pp. 925-48.

Zhaung ZP, McCauley R. Ubiquitin is involved in the in vitro insertion of monoamine oxidase $B$ into mitochondrial outer membranes. J Bio Chem 1989;264:14594-6. 
Y.H. Huang et al. 